\title{
Physical exercise for bone health in men with prostate cancer receiving androgen deprivation therapy: a systematic review
}

\author{
Barbara Bressi ${ }^{1,2}$ (D) $\cdot$ Maribel Cagliari ${ }^{3} \cdot$ Massimiliano Contesini $^{4} \cdot$ Elisa Mazzini ${ }^{5}$. \\ Franco Antonio Mario Bergamaschi ${ }^{6}$. Alfredo Moscato ${ }^{6} \cdot$ Maria Chiara Bassi $^{7} \cdot$ Stefania Costi $^{8,3}$
}

Received: 7 July 2020 / Accepted: 13 October 2020 / Published online: 29 October 2020

(C) The Author(s) 2020

\begin{abstract}
Purpose Androgen deprivation therapy (ADT) is a treatment used in men with prostate cancer (PCa); however it is responsible for many adverse effects, with negative impact on quality of life. ADT causes loss of bone mineral density (BMD) and skeletal muscle mass, alteration of body composition, and cognitive function, which altogether lead to increased risk of accidental falls and fractures. This systematic review analyses the effectiveness of physical exercise (PE) in preventing accidental falls and fractures and reducing the loss of BMD in men with PCa receiving ADT.

Methods We searched MEDLINE, EMBASE, CINAHL, and the Cochrane Library for articles between database inception and September 2, 2020. Eligible studies included randomized controlled trials (RCTs) investigating the effects of exercise on bone health in men with PCa receiving ADT.

Results Nine RCTs were included. Experimental PE consisted in multicomponent programmes that involved aerobic, resistance, impact-loading exercise, and football training. None of the RCTs investigated the risk of accidental falls and fractures, while two trials reported beneficial effects of PE on lumbar spine, hip, and femoral shaft BMD. No further significant difference was detected in the outcomes investigated.

Conclusion Evidence of the effectiveness of PE to prevent the risk of accidental falls and fractures and BMD loss is lacking. Nevertheless, clinical guidelines recommend PE as a part of the clinical management of men with PCa receiving ADT due to its known numerous health benefits. Research should focus on PE strategies to prevent accidental falls, a clinically relevant outcome in this vulnerable population.

Trial registration The study protocol was registered with International Prospective Register of Systematic Reviews (PROSPERO, number CRD 42020158444) on 04/28/2020.
\end{abstract}

Keywords Prostatic neoplasms $\cdot$ Exercise $\cdot$ Accidental falls $\cdot$ Fractures, bone $\cdot$ Physical therapy modalities $\cdot$ Prevention

Electronic supplementary material The online version of this article (https://doi.org/10.1007/s00520-020-05830-1) contains supplementary material, which is available to authorized users.

Barbara Bressi

barbara.bressi@ausl.re.it

$1 \quad \mathrm{PhD}$ Program in Clinical and Experimental Medicine, Department of Biomedical, Metabolic and Neural Sciences, University of Modena and Reggio Emilia, Reggio Emilia, Italy

2 Physical Medicine and Rehabilitation Unit, Azienda USL-IRCCS di Reggio Emilia, Reggio Emilia, Italy

3 Department of Surgery, Medicine, Dentistry and Morphological Sciences, University of Modena and Reggio Emilia, Modena, Italy

4 Human Resource Development - Training Radiographers and Radiations Terapist, Azienda USL-IRCCS di Reggio Emilia, Reggio Emilia, Italy
5 Medical Directorate Hospital Network, Azienda USL-IRCCS di Reggio Emilia, Reggio Emilia, Italy

6 Urology and Mininvasive Surgery, Department of General and Specialist Surgeries, Azienda USL-IRCCS di Reggio Emilia, Reggio Emilia, Italy

7 Medical Library, Azienda USL-IRCCS di Reggio Emilia, Reggio Emilia, Italy

8 Scientific Directorate, Azienda USL-IRCCS di Reggio Emilia, Reggio Emilia, Italy 


\section{Introduction}

Prostate cancer $(\mathrm{PCa})$ is the most prevalent cancer among men worldwide, with 3.724 .658 cases in 2018 [1].

Androgen deprivation therapy (ADT) is currently the standard systemic treatment in patients with metastatic or more aggressive $\mathrm{PCa}$ [2]. Often, ADT is used in combination with radiotherapy for localized advanced $\mathrm{PCa}$ with the aim to increase survival and control disease progression [2].

However, ADT is responsible for many adverse effects, with negative impact on quality of life [3]. Apart from the increased risk for cardiovascular events and metabolic syndrome $[4,5]$, ADT also alters the body composition, with loss in skeletal muscle mass that leads to a decrease in muscle strength $[6,7]$. Also, patients on ADT manifest significant loss of bone mineral density (BMD), which occurs especially within the first year of treatment [8] and is associated with higher osteoporosis rates and risk of fractures [9]. Moreover, ADT seems responsible for cognitive dysfunction, although this finding has not been completely clarified and needs further investigation [10].

It is known that both reduction in muscle strength and cognitive dysfunction are predictors of higher fall rates and hospitalization in older adults [11, 12]. Thus, considering the loss of BMD, altogether these side effects of ADT explain the increased risk of accidental falls and fractures in this population [13].

In elderly adults, physical exercise (PE) has been proposed in different modalities as a strategy to produce several health benefits [14]. Recent guidelines addressing elderly adults recommend multicomponent exercise programmes, including resistance and neuromotor exercises, as a strategy to reduce the risk of accidental falls [14] as PE can prevent osteoporosis and improves body composition, muscle strength, and cognitive function [14]. Moderate-vigorous intensity programmes that include balance exercises seem to be particularly effective to reduce the risk of accidental falls [14].

Initial evidence indicates that in patients with cancer, $\mathrm{PE}$ may produce numerous benefits on physical performance, quality of life, and cancer-related fatigue [15]. In patients with PCa receiving ADT, exercise is beneficial to body composition, muscle strength, and physical performance [16-18], while its effects on bone health, cardiometabolic risk, quality of life, and cognitive functions remain uncertain [19, 20].

Patients receiving ADT are more exposed to the risk of accidental falls and fractures [9] due to the side effects of this drugs. As PE is recommended in healthy elderly adults to prevent these risks and also to prevent bone loss, we hypothesized that PE could be effective in preventing accidental falls and fractures even in men with $\mathrm{PCa}$ receiving ADT. Furthermore, recent evidence suggests PE as strategy to prevent osteoporosis in men receiving ADT when associated with pharmacological therapy [21]. Thus, we conducted this systematic review to search for evidence of the effectiveness of exercise on bone health in this population. Specifically, we searched for randomized controlled trials that implemented PE programmes to prevent accidental falls and fractures and/or to prevent the loss of BMD, in patients with $\mathrm{PCa}$ treated with ADT.

\section{Methods}

This systematic review followed the Preferred Reporting Items for Systematic Reviews and Meta-analyses (PRISMA) guidelines [22]. The study protocol was registered with International Prospective Register of Systematic Reviews (PROSPERO, number CRD 42020158444).

\section{Search strategy and selection criteria}

A systematic review of the literature was performed through sequential, individualized searches in MEDLINE, EMBASE, CINAHL, and the Cochrane Library. We searched for studies published up to September 2, 2020, without filters for study design or language. The search terms and strategies used are reported in online resource 1. Duplicates were removed in EndNote (version X7.5). Also, we performed a manual search in the reference lists of the studies included in this review to find any other relevant citation that may have been missed by the electronic search.

We included randomized controlled trials (RCTs) investigating the effects of supervised or unsupervised exercise on bone health in adult individuals with PCa receiving ADT.

Studies were eligible if the experimental intervention consisted of structured PE programmes compared with standard care or placebo active control. When exercise was associated with dietary supplements, studies were included if the exercise was clearly the predominant part of the experimental intervention. Furthermore, studies were eligible if they investigated the number of accidental falls or fractures that occurred in a specific timeframe or if they reported data on bone density by dualenergy X-ray absorptiometry (DEXA). Studies focusing on generalized advice and education on the benefit of exercise or studies that collected data on pathological (and not accidental) fractures were excluded.

\section{Data analysis}

Two investigators (B.B., M.C.) screened the title and abstract of all the citations retrieved to check their appropriateness related to the purpose of this review. The investigators also retrieved and checked for eligibility the full texts of studies deemed appropriate. Then, two investigators (B.B., S.C.) assessed the eligible studies for their methodological quality according to the 
Cochrane risk-of-bias tool [23]. In the whole process, any disagreement was resolved by discussion and consensus.

Two investigators (B.B., M.C.) extracted the following data from studies included: authors, year and country, sample size and average age, exclusion criteria, bone outcome measures collected and follow-up duration, general characteristics of the experimental intervention and standard care, drop-out rate. When essential data were missing, the investigators requested them from authors (at least three attempts).

\section{Results}

\section{Bibliographic search results}

The electronic search yielded 304 citations, duplications excluded. One more citation was retrieved through the manual search, for a total of 305. According to the screening of title and abstract, 269 citations were excluded because they did not focus on the topic under investigation.

Thirty-six full texts were reviewed for eligibility, 27 of which were excluded for the following reasons: three conference abstracts and six study protocols referred to published full texts already retrieved [24-31]; four studies did not meet inclusion criteria with respect to the outcome, as one measured only pathological fractures [32], and the others did not report data on bone health $[31,33,34]$; one study did not test a structured physical exercise intervention, focusing instead on patient education [35]; two studies compared different structured physical exercise interventions, without comparison to standard care [36, 37]. Finally, eleven studies were also excluded since they reported insufficient data for analysis [38] or were protocols of ongoing studies [39-48]. We contacted the corresponding authors in order to obtain preliminary results (minimum three attempts), but the ones who replied said they had no data to share yet.

Thus, nine published full texts met the inclusion criteria and contributed their data to this review [24-30, 49, 50]. These full texts accounted for eight study designs, as the two by Uth et al. $[28,29]$ reported data collected at the 3- and 8month follow-up, respectively, of the same study design and sample (Fig. 1). Of note, the study by Bjerre et al. [30] included patients with PCa regardless of their treatment with ADT. However, they reported specific data for the subgroup of patients on ADT and these data were considered in this review.

\section{Risk of bias of the included studies}

The Cochrane risk-of-bias analysis of the included studies is reported in Fig. 2. Two studies did not report sufficient information to assess the adequacy of the random sequence generation $[28,29,49,51]$. Due to the nature of the intervention, seven of the nine included studies did not provide blinding to group assignment for both participants and personnel [24-30, 49]. Moreover, four studies did not report enough information to judge blinding of outcome [24-26, 49]. Nevertheless, all the included studies were judged at low risk of detection bias since outcome measures were frequently objective. The analytical assessment of the risk of bias for each study included is reported in online resource 2 .

\section{Study characteristics}

Table 1 summarizes the characteristics of the studies included in this review. All were RCTs published in the last decade in different continents [24-30, 49, 50]. All studies allowed for the recruitment of patients treated at several specialized hospitals.

One study was a three-armed RCT comparing two active interventions with one control [25]. For the purposes of this review, we considered both the comparisons. Two studies were cross-over designs, and, for this review, we considered data of the first follow-up, before the cross-over, which was scheduled at 6 months for both studies $[25,26]$.

A high drop-out rate $(\geq 28 \%)$ was registered in two studies [29, 49] and equal to $20 \%$ in a further one [50], whereas one study did not report this data point for the subgroup of patients receiving ADT [30]. The safety of interventions was assessed by recording the number and type of adverse events. Only two trials reported adverse events related to exercise [27, 28]: one partial Achilles tendon rupture [28], two fibula fractures [28], and five minor musculoskeletal injuries [27, 28]. Other trials registered generic health issues not related to the PE intervention that occurred both in the experimental and in the control group, such as hospitalization $(n=22)[25-27,29,49,50]$, injury/accident $(n=10)$ [25-27], death $(n=4)$ [25, 49], and others $(n=4)$ [24, 26-29]. Bjerre et al. [30] analysed safety outcomes as number of falls, fractures, and serious adverse events occurring even in the subgroup of patients receiving ADT (respectively $n=5, n=1$, and $n=4$ ), without any further detailed classification.

\section{Participants}

The sample size of the eight RCTs selected in this review ranged from 51 to 154 individuals, for a total of 625 participants, of whom 351 were randomized to receive experimental $\mathrm{PE}$ and 274 were randomized to receive standard care. The sample was made up of males aged from 66.0 to 70.8 years with local or metastatic PCa receiving ADT.

All the study designs excluded patients with restrictions to PE based on specific assessments (e.g., inability to walk $400 \mathrm{~m}$ [24-26], $\mathrm{VO}^{2} \max <35 \mathrm{ml} / \mathrm{kg} / \mathrm{min}[28,29]$, pain in the metastatic site associated to activity $[28,29])$ or based on clinicians' judgement [24-27, 30, 49, 50]. Five studies also excluded patients with musculoskeletal, cardiovascular, and 
Fig. 1 PRISMA flow chart of search and study selection process
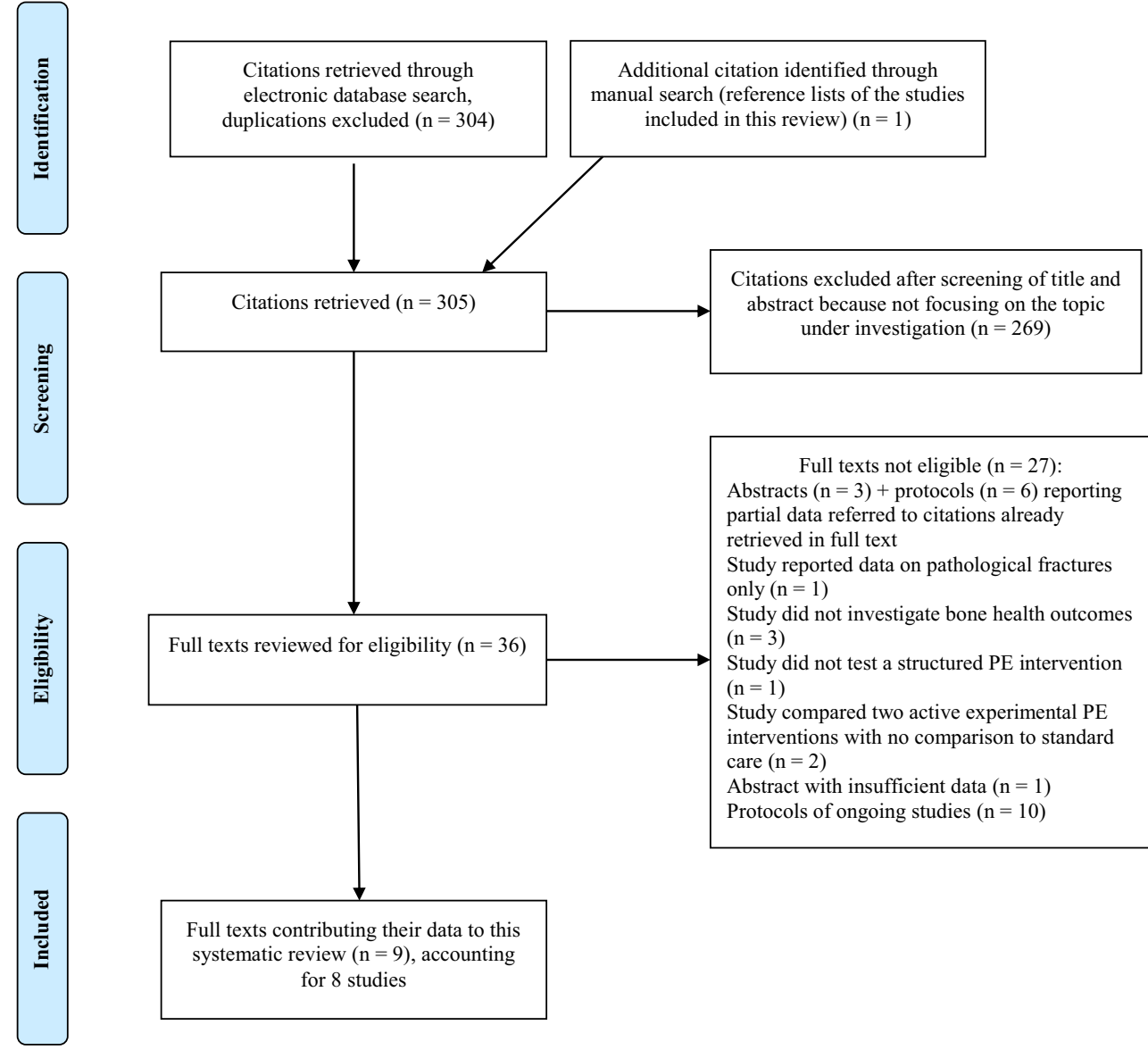

Full texts not eligible $(\mathrm{n}=27)$ : Abstracts $(\mathrm{n}=3)+$ protocols $(\mathrm{n}=6)$ reporting partial data referred to citations already retrieved in full text

Study reported data on pathological fractures only $(\mathrm{n}=1)$

Study did not investigate bone health outcomes $(n=3)$

Study did not test a structured PE intervention $(\mathrm{n}=1)$

Study compared two active experimental PE interventions with no comparison to standard care $(\mathrm{n}=2)$

Abstract with insufficient data $(\mathrm{n}=1)$

Protocols of ongoing studies $(n=10)$ neurological disorders that could inhibit them from exercising [24-29] or patients with contraindications to unsupervised exercise [50]. Further exclusion criteria have been summarized in detail in Table 1.

\section{Characteristics of control group intervention and the experimental group intervention}

Table 2 summarizes the characteristics of standard care and experimental PE programmes.

The control group intervention consisted of stretching activities $[49,50]$ or educational material $[25,30,50]$ or simply in encouraging patients to maintain their usual level of physical activity [27-29]. Taaffe et al. [26] provided all participants with standard daily supplementation of calcium (1000 mg/day) and vitamin D3 (800 IU/day).

Experimental PE interventions were characterized by multicomponent programmes [24-26, 28-30, 49, 50], with only one exception that implemented a single component of PE [27]. In most cases, PE consisted in aerobic exercise (AE) that could also be performed as weight-bearing activities and that was associated with resistance exercise (RE) [24-26, 50] and with impact-loading exercise (IE) [26]. Two study designs implemented football training (FT) as experimental PE
[28-30]; although the full texts did not report this type of training in detail, it is likely that, by its nature, it included $\mathrm{AE}$ and IE, among others (e.g., RE, stretching).

Most experimental interventions were performed in 1-hour sessions repeated two or three times a week [24-30, 49]. Most of the studies described how the PE components were progressively modulated in terms of intensity, volume, and type of exercise [24-27, 49, 50]. Although the intensity of FT was not defined, this type of intervention was implemented through an initial warm-up followed by 2 matches lasting $15 / 20 \mathrm{~min}[28-30]$. Uth et al. [28, 29] progressively increased the number of matches and the frequency of sessions per week.

Experimental PE were implemented as supervised exercise in clinics [24-26] or in sports facilities [28-30], as a combination of supervised and unsupervised (home-based) sessions $[25,27,49]$, or as unsupervised home-based PE only [50].

\section{Bone outcomes}

\section{Accidental falls and fractures}

None of the studies selected for this review was designed to analyse the risk of accidental falls and/or fractures as an 


\begin{tabular}{|c|c|c|c|c|c|c|c|}
\hline \multicolumn{8}{|c|}{ Risk-of-bias analysis of RCTs included } \\
\hline & 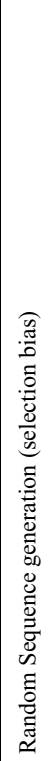 & 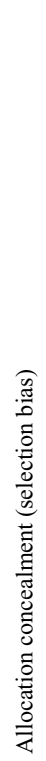 & 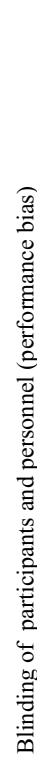 & 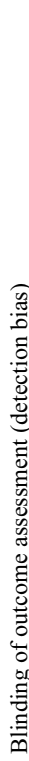 & 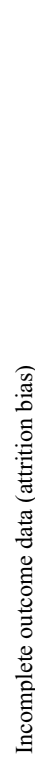 & 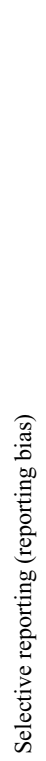 & 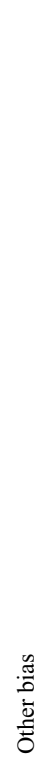 \\
\hline Cormie et al. (2015) & + & & & & & & $1+$ \\
\hline Newton et al. (2019) & & & & & & & \\
\hline Taaffe et al. $(2019)^{26}$ & + & & & & & + & \\
\hline Nilsen et al. $(2015)^{27}$ & + & & & & & & \\
\hline $\begin{array}{l}\text { Uth et al. (2013, } \\
2016)^{28,29,51}\end{array}$ & $?$ & & & & & & \\
\hline Bjerre et al. $(2019)^{30}$ & + & & & & & & \\
\hline $\begin{array}{l}\text { Winters-Stone et al. } \\
(2014)^{49}\end{array}$ & $?$ & & & & & & \\
\hline Kim et al. $(2018)^{50}$ & P & & & & & & \\
\hline
\end{tabular}

Fig. 2 Risk-of-bias analysis of RCTs included

outcome measure of the effectiveness of experimental PE in reducing those risks. All study designs measured adverse events $[24-30,49,50]$. The two studies that tested FT also recorded fractures occurring during this kind of intervention to judge its safety due to the increased risk of collisions with other players and falls [28-30]. Although this was not the outcome we were interested in, we underline that all the experimented PE programmes were deemed safe [24-30, 49, 50].

Of note, several studies secondarily collected data on physical function through heterogeneous tests (e.g., Flamingo balance test, sit-to-stand test, etc.) $[24,27-29,50]$. The proof of effectiveness of experimental PE was demonstrated through the sit-to-stand test $[24,27,50]$, which is valid to measure muscle power of the lower limbs; its validity in predicting accidental falls and fractures in patients with cancer, however, must still be demonstrated [52].

\section{BMD}

Table 3 reports the results of between-group comparisons of $\mathrm{BMD}$ at the various anatomical sites.

All the RCTs selected for this review reported data on bone density measured by DEXA at different anatomical sites (Table 3). Lumbar spine BMD was collected in all the included studies, while femoral neck BMD was analysed in seven of them $[24,25,27,29,30,49,50]$. At the 6-month follow-up, Newton et al. [25] recorded a significant difference between groups for lumbar spine BMD (mean change $0.014 \mathrm{~g} / \mathrm{cm}^{2}$, $95 \%$ CI $0.001-0.027, p=0.039)$ and a positive trend for femoral neck BMD (mean change $0.010 \mathrm{~g} / \mathrm{cm}^{2}, 95 \%$ CI 0.000 $0.020, p=0.050$ ), in favour of the experimental resistance and impact-loading PE compared with control group. No further significant difference was detected by any of the studies in these outcome measures. Of note, a per-protocol analysis performed by Winters-Stone et al. [49] at the level of single lumbar vertebra reported a significant difference in BMD only for $\mathrm{L}_{4}(p=0.03)$, in favour of experimental PE.

Total hip BMD was measured by seven study designs [25-27, 29, 30, 49, 50], with significant differences recorded only by Uth et al. [29] on both hips at the 8-month follow-up (right $0.015 \mathrm{~g} / \mathrm{cm}^{2}, 95 \%$ CI $0.003-0.027, p=0.015$; left $0.017 \mathrm{~g} / \mathrm{cm}^{2}, 95 \%$ CI $0.002-0.032, p=0.030$ ). This study was the only one that collected data on BMD at the femoral shaft of both legs, recording a difference in favour of experimental PE on both sides (right $0.018 \mathrm{~g} / \mathrm{cm}^{2}, 95 \%$ CI 0.004 $0.032, p=0.016$; left $0.024 \mathrm{~g} / \mathrm{cm}^{2}, 95 \%$ CI $0.005-0.044, p=$ $0.015)$ [29].

No further statistically significant difference was registered for BMD at any further anatomical site examined, such as whole body [24-28, 30], trochanter [25, 27, 49], or legs [28].

Considering the almost total absence of data in favour of PE with respect to this outcome, which was collected in various anatomical sites, we deemed it inappropriate to carry out a meta-analysis.

\section{Further results: bone turnover markers}

Six study designs also assessed several bone turnover markers (BTMs) such as markers of bone formation (alkaline phosphatase, procollagen type 1 amino-terminal propeptide and osteocalcin) or markers of bone resorption (C-terminal telopeptide of type I collagen, N-terminal telopeptide of type I collagen) [24-26, 28, 29, 49, 50].

At the 3-month follow-up, Uth et al. [28, 29] registered a statistically significant difference in favour of experimental PE for the markers of bone formation procollagen type 1 


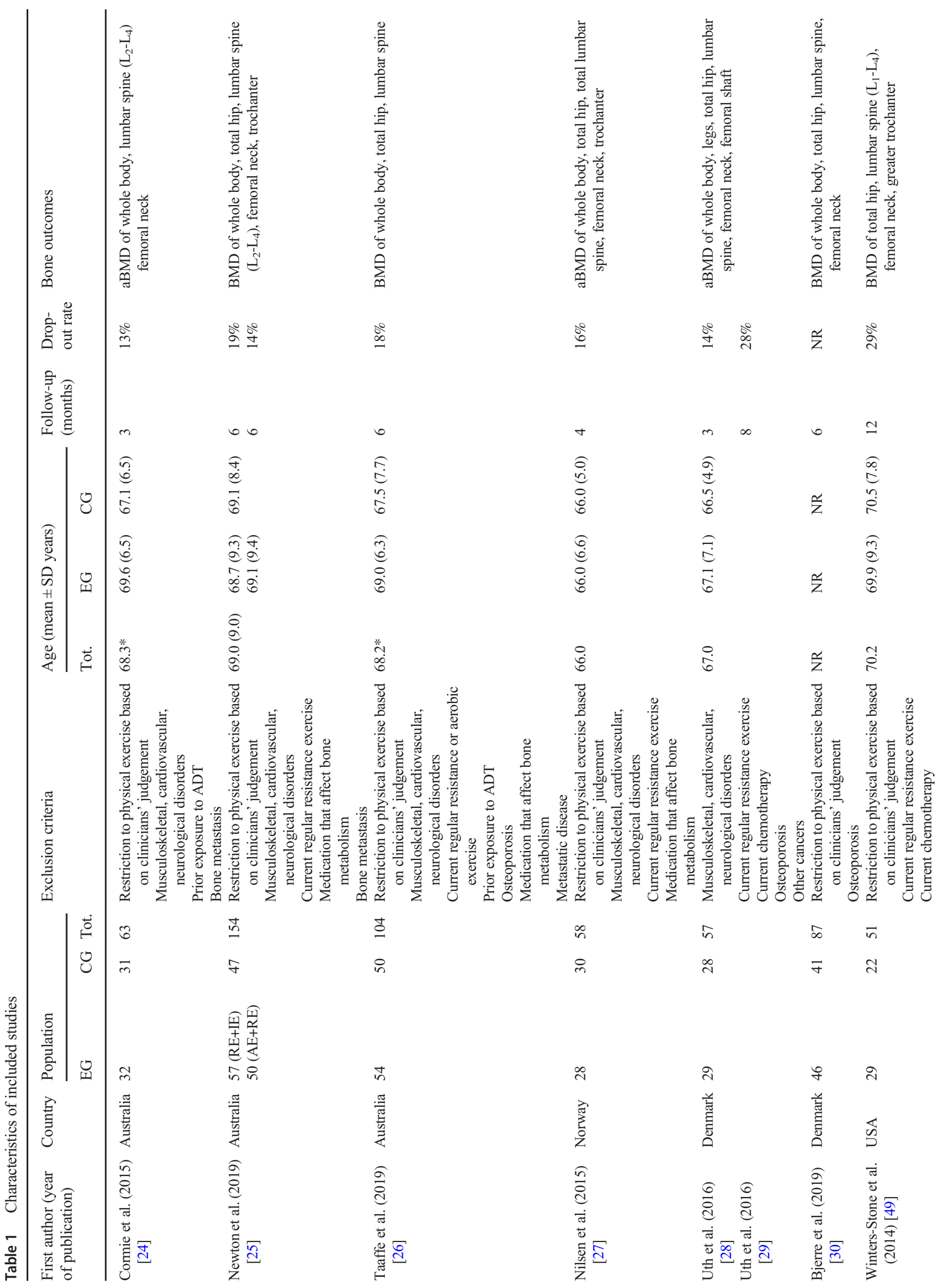




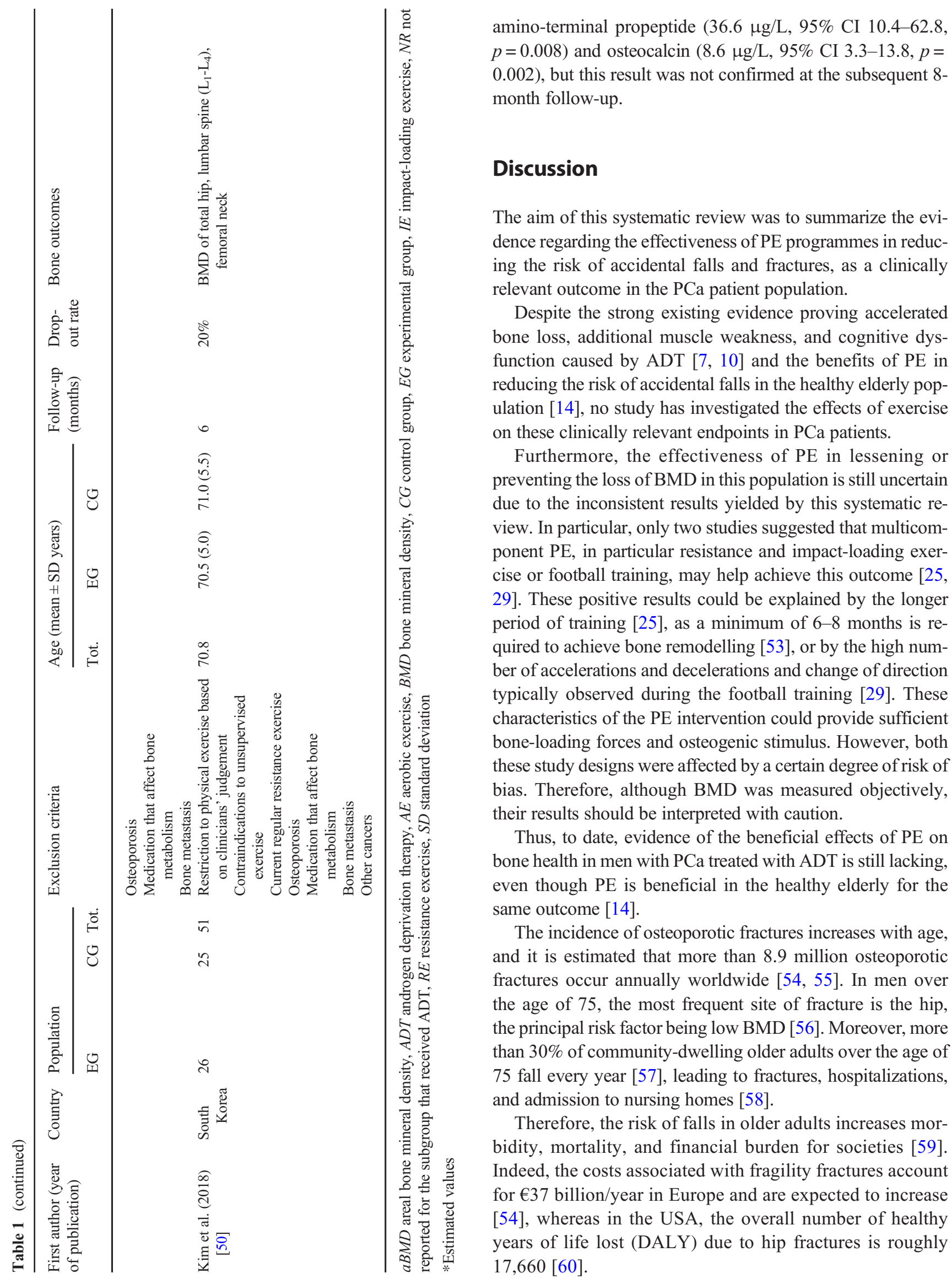




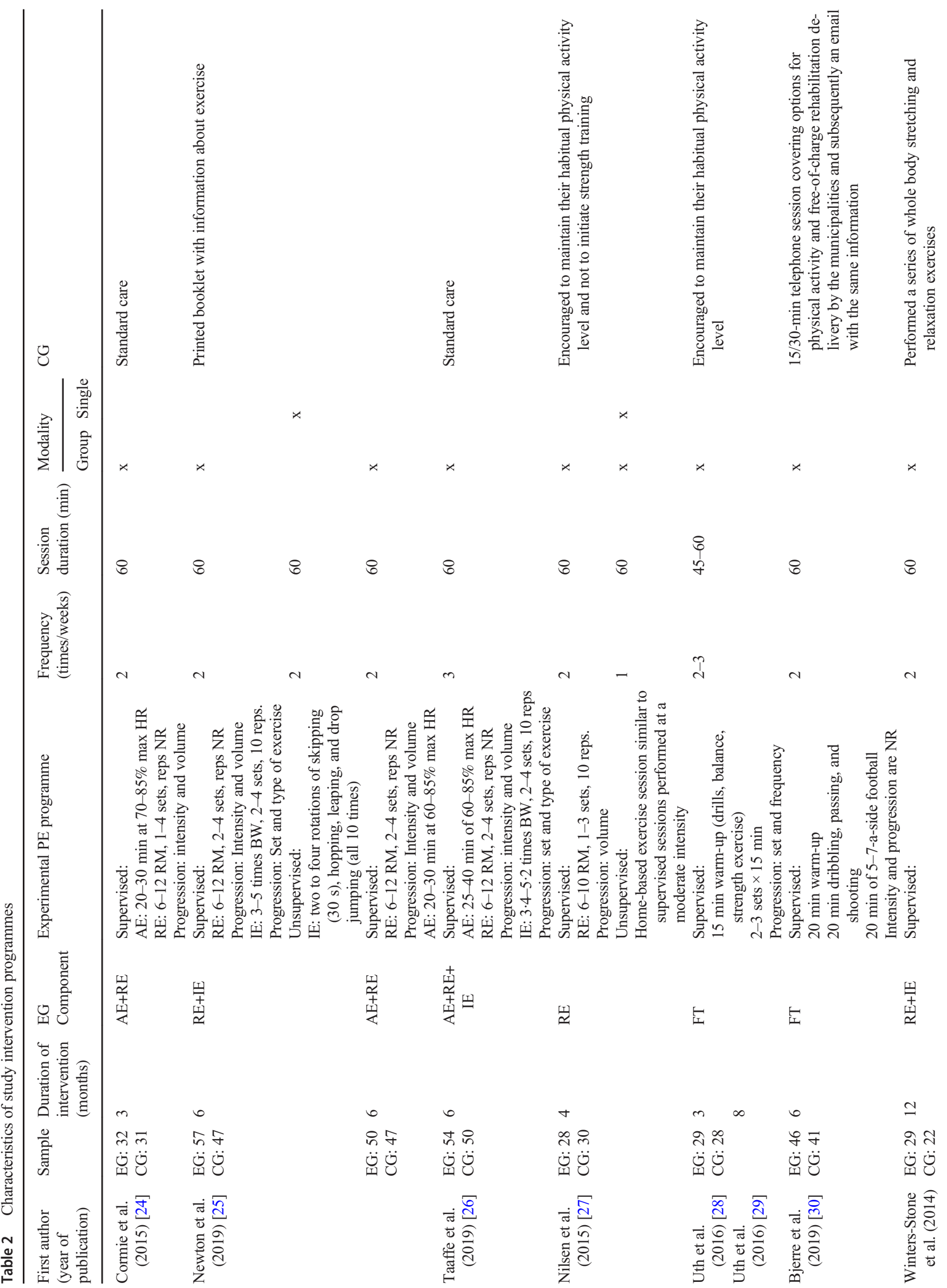




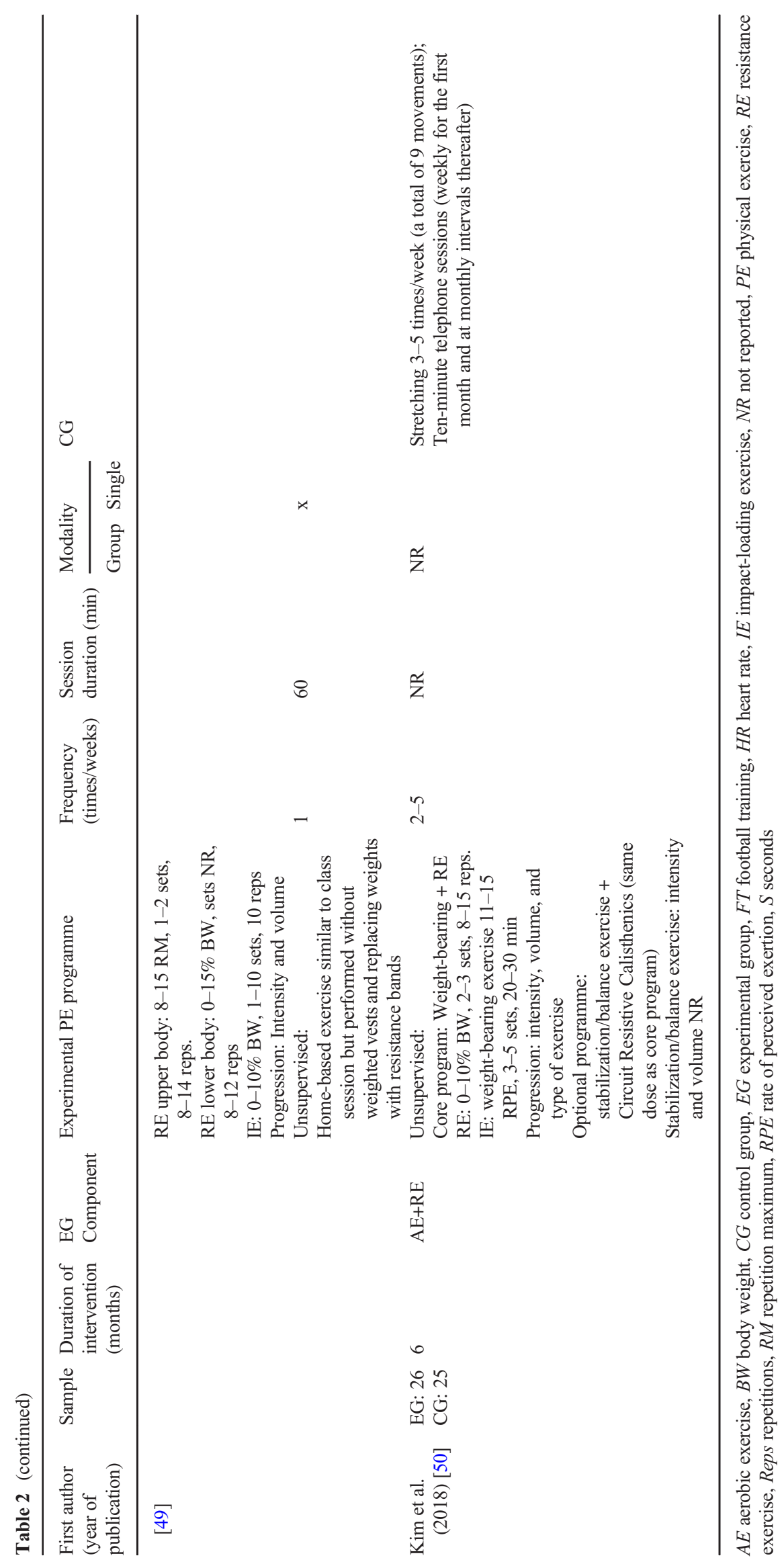


Table 3 Between-group comparisons for BMD

\begin{tabular}{|c|c|c|c|c|c|}
\hline & Follow-up (months) & BMD outcome & Mean change between groups $\left(\mathrm{g} / \mathrm{cm}^{2}\right)$ & $95 \% \mathrm{CI}$ & $p$ value \\
\hline \multirow[t]{3}{*}{ Cormie et al. (2015) [24] } & \multirow[t]{3}{*}{3} & Whole body & $-0.002 *$ & -0.013 to 0.009 & 0.692 \\
\hline & & Lumbar spine & $-0.009 *$ & -0.029 to 0.012 & 0.410 \\
\hline & & Femoral neck & $0.000 *$ & -0.025 to 0.024 & 0.987 \\
\hline \multirow[t]{10}{*}{ Newton et al. (2019) [25] } & \multirow[t]{5}{*}{$6(\mathrm{RE}+\mathrm{IE})$} & Whole body & $0.005^{*}$ & -0.002 to 0.011 & 0.174 \\
\hline & & Total hip & $0.007 *$ & -0.002 to 0.016 & 0.128 \\
\hline & & Lumbar spine & $0.014 *$ & 0.001 to 0.027 & 0.039 \\
\hline & & Femoral neck & $0.010^{*}$ & 0.000 to 0.020 & 0.050 \\
\hline & & Trochanter & $-0.003 *$ & -0.010 to 0.004 & 0.449 \\
\hline & \multirow[t]{5}{*}{$6(\mathrm{AE}+\mathrm{RE})$} & Whole body & $0.003 *$ & -0.007 to 0.0012 & 0.614 \\
\hline & & Total hip & $0.001 *$ & -0.009 to 0.011 & 0.807 \\
\hline & & Lumbar spine & $0.004 *$ & -0.009 to 0.017 & 0.525 \\
\hline & & Femoral neck & $-0.003 *$ & -0.014 to 0.008 & 0.571 \\
\hline & & Trochanter & $-0.002 *$ & -0.01 to 0.007 & 0.699 \\
\hline \multirow[t]{3}{*}{ Taaffe et al. (2019) [26] } & \multirow[t]{3}{*}{6} & Whole body & NR & NR & 0.827 \\
\hline & & Total hip & NR & NR & 0.848 \\
\hline & & Lumbar spine & NR & NR & 0.111 \\
\hline \multirow[t]{5}{*}{ Nilsen et al. (2015) [27] } & \multirow[t]{5}{*}{4} & Whole body & $0.00 *$ & -0.02 to 0.01 & 0.520 \\
\hline & & Total hip & $0.00 *$ & -0.01 to 0.01 & 0.690 \\
\hline & & Total lumbar spine & $0.00 *$ & -0.02 to 0.01 & 0.847 \\
\hline & & Femoral neck & $0.00 *$ & -0.02 to 0.01 & 0.467 \\
\hline & & Trochanter & $0.00 *$ & -0.01 to 0.00 & 0.221 \\
\hline \multirow[t]{2}{*}{ Uth et al. (2016) [28] } & \multirow[t]{2}{*}{3} & Whole body & 0.01 & -0.00 to 0.01 & 0.188 \\
\hline & & Legs & 0.00 & -0.00 to 0.01 & 0.336 \\
\hline \multirow[t]{7}{*}{ Uth et al. (2016) [29] } & \multirow[t]{7}{*}{8} & Total hip & R: 0.015 & 0.003 to 0.027 & 0.015 \\
\hline & & & L: 0.017 & 0.002 to 0.032 & 0.030 \\
\hline & & Lumbar spine & 0.028 & -0.010 to 0.065 & 0.144 \\
\hline & & Femoral neck & R: 0.015 & -0.002 to 0.031 & 0.078 \\
\hline & & & $\mathrm{L}: 0.015$ & -0.01 to 0.032 & 0.072 \\
\hline & & Femoral shaft & R: 0.018 & 0.004 to 0.032 & 0.016 \\
\hline & & & L: 0.024 & 0.005 to 0.044 & 0.015 \\
\hline \multirow[t]{4}{*}{ Bjerre et al. (2019) [30] } & \multirow[t]{4}{*}{6} & Whole body & 0.005 & -0.007 to 0.017 & 0.40 \\
\hline & & Total hip & $-0.009 *$ & -0.033 to 0.014 & 0.43 \\
\hline & & Lumbar spine & $0.0017 *$ & -0.019 to 0.053 & 0.34 \\
\hline & & Femoral neck & $0.007 *$ & -0.009 to 0.023 & 0.39 \\
\hline \multirow[t]{4}{*}{ Winters-Stone et al. (2014) [49] } & \multirow[t]{4}{*}{12} & Total hip & NR & NR & 0.37 \\
\hline & & Lumbar spine & NR & NR & 0.47 \\
\hline & & Femoral neck & NR & NR & 0.77 \\
\hline & & Greater trochanter & NR & NR & 0.58 \\
\hline \multirow[t]{3}{*}{ Kim et al. (2018) [50] } & \multirow[t]{3}{*}{6} & Total hip & NR & NR & 0.727 \\
\hline & & Lumbar spine & NR & NR & 0.756 \\
\hline & & Femoral neck & NR & NR & 0.888 \\
\hline
\end{tabular}

$A E$ aerobic exercise, $B M D$ bone mineral density, $C I$ confidence intervals, $I E$ impact-loading exercise, $L$ left, $N R$ not reported, $R$ right, $R E$ resistance exercise

*Analyses adjusted for baseline values

Considering the progressive aging of the population, recent guidelines suggest that future research should identify individuals at increased risk of fracture, to whom fracture prevention strategies should be targeted [61] in order to contain the increase in costs associated with this event [62]. We think that patients with $\mathrm{PCa}$ receiving ADT are among those individuals because the side effects they have affect bone and lead to double the healthcare cost per person [63]. Moreover, as fall prevention programmes are recommended to the elderly in general, as community-dwelling adults with cancer have 
greater accidental fall rates than do healthy elderly individuals and as patients undergoing active treatment are even more at risk [64], it seems logical to expect that the beneficial effects of PE would be greater in patients with PCa receiving ADT compared with healthy elderly individuals.

However, this systematic review demonstrated an almost complete lack of studies supporting this. Not only have clinically relevant outcomes never been investigated but also few of the RCTs included were powered to detect the effects of PE on BMD $[25,26,49,50]$.

We must say, however, that our review was limited to collecting evidence on PE. As we did not consider other types of interventions, such as nutritional and educational programmes that could help to prevent accidental falls and fractures, we cannot rule out that an evidence-based intervention different from stand-alone PE could be successfully applied. Furthermore, ten protocols of ongoing studies were retrieved by our search strategy; it is therefore very likely that in the next few years the conclusion drawn today, thanks to this extensive review conducted with rigorous methodology, will be outdated.

A final consideration regards the type of PE programmes tested in the studies included in this review: most combined different exercise modalities, such as resistance, weightbearing endurance, and impact loading exercises, as recommended to provide benefits to bone health [53]. Moreover, PE programmes were of moderate-high intensity in all cases, suggesting that, according to the evidence and expertise, low-impact exercise may have no effect on BMD [53]. Despite this, the PE programmes did not produce the desired result on bone mass. This may be due to the insufficient power of some of the included studies, or it may be due to poor adherence to treatment, which is always an issue in studies involving lifestyle changes [65]. Poor adherence to treatment means that the expected dose of exercise is not achieved by participants. Thus, it can be difficult to determine the effect of the exercise on the outcomes of interest. It could also be that, however intense the programme, PE may not be sufficient to counteract the loss of bone mass induced by aging and ADT.

Thus, to conclude, experts recommend exercise as part of the treatment regimen of patients with cancer thanks to its large number of health benefits [19]. This review suggests that there is still no strong evidence to support this choice to prevent bone density loss in patients with PCa receiving ADT, according to recent literature $[16,21]$. However, since exercise is an effective strategy to produce a large number of health benefits, future research should investigate the effects of PE to prevent the risk of accidental falls in this population, which is a clinically relevant outcome. For this purpose, PE should include coordination and balance exercises as well as muscle-strengthening activities. Evidence is needed regarding more precise training components, dose, and progression of exercise to prevent falls.
Acknowledgements We thank Jacqueline M. Costa for the English language editing.

Author contributions Barbara Bressi, Maribel Cagliari and Stefania Costi contributed to the conception and design of the work, literature search, selection of the trials, assessment of trial quality and data collection and contributed to the interpretation of data. They also drafted the work and approved the final version to be published. Massimiliano Contesini, Elisa Mazzini, Franco Antonio Mario Bergamaschi and Alfredo Moscato contributed to the conception and design of the work and to the interpretation of data. They revised the draft of the manuscript and approved the final version to be published. Maria Chiara Bassi contributed to the conception and design of work, the literature search and the selection of the trials and contributed to the interpretation of data. She also drafted the work and approved the final version to be published.

\section{Compliance with ethical standards}

Conflict of interest The authors declare that they have no conflict of interest.

Ethical approval No ethical approval was required since we used only published data.

Consent to participate Not applicable.

Consent to publication Not applicable.

Availability of data and material No additional data available. Only published data were used. The corresponding author had full access to all the data in the study and had final responsibility for the decision to submit for publication.

Code availability Not applicable.

Open Access This article is licensed under a Creative Commons Attribution 4.0 International License, which permits use, sharing, adaptation, distribution and reproduction in any medium or format, as long as you give appropriate credit to the original author(s) and the source, provide a link to the Creative Commons licence, and indicate if changes were made. The images or other third party material in this article are included in the article's Creative Commons licence, unless indicated otherwise in a credit line to the material. If material is not included in the article's Creative Commons licence and your intended use is not permitted by statutory regulation or exceeds the permitted use, you will need to obtain permission directly from the copyright holder. To view a copy of this licence, visit http://creativecommons.org/licenses/by/4.0/.

\section{References}

1. World Health Organization. International Agency for Research on Cancer (2018) Cancer Today. https://gco.iarc.fr/today/home. Accessed 2 Sept 2020

2. Mohler JL, Kantoff PW, Armstrong AJ, Bahnson RR, Cohen M, D'Amico AV, Eastham JA, Enke CA, Farrington TA, Higano CS, Horwitz EM, Kawachi MH, Kuettel M, Lee RJ, MacVicar GR, Malcolm AW, Miller D, Plimack ER, Pow-Sang JM, Richey S, Roach M III, Rohren E, Rosenfeld S, Small EJ, Srinivas S, Stein C, Strope SA, Tward J, Walsh PC, Shead DA, Ho M (2013) Prostate cancer, version 1.2014: Featured updates to the NCCN 
Guidelines. J Natl Compr Canc Netw 11:1471-1479. https:/doi. org/10.6004/jncen.2013.0174

3. Alibhai SMH, Gogov S, Allibhai Z (2006) Long-term side effects of androgen deprivation therapy in men with non-metastatic prostate cancer: a systematic literature review. Crit Rev Oncol Hematol 60:201-215. https://doi.org/10.1016/j.critrevonc.2006.06.006

4. Carneiro A, Sasse AD, Wagner AA, Peixoto G, Kataguiri A, Neto AS, Bianco BAV, Chang P, Pompeo ACL, Tobias-Machado M (2014) Cardiovascular events associated with androgen deprivation therapy in patients with prostate cancer: a systematic review and meta-analysis. World J Urol 33:1281-1289. https://doi.org/10. 1007/s00345-014-1439-6

5. Morote J, Gómez-Caamaño A, Alvarez-Ossorio JL, Pesqueira D, Tabernero A, Gómez Veiga F, Lorente JA, Porras M, Lobato JJ, Ribal MJ, Planas J, ANAMET Investigators Group José M Saladié, Gemma Sancho, Humberto Villavicencio, José Segarra, José Comet, José Francisco Suárez Dr., M José Ribal Dr., José Antonio Llorente Dr., Juan Uría Dr., Jesús Guajardo Dr., Antonio Gómez Caamaño Dr., Camilo García Freire Dr., Antonio Ojea Dr., Juan Mata Dr., M ${ }^{\mathrm{a}}$ Luisa Vázquez Dr., Francisco Gómez Veiga Dr., Daniel Pesqueira Dr., Juan Pablo Ciria Dr., Roberto Llarena Dr., Jesús Miguel Unda Dr., Angel José Tabernero Dr., Angel Silmi Dr., Carlos Hernández Dr., Alfredo R (2015) The metabolic syndrome and its components in patients with prostate cancer on androgen deprivation therapy. J Urol 193:1963-1969. https://doi.org/10. 1016/j.juro.2014.12.086

6. Haseen F, Murray LJ, Cardwell CR, O'Sullivan JM, Cantwell MM (2010) The effect of androgen deprivation therapy on body composition in men with prostate cancer: systematic review and metaanalysis. J Cancer Surviv 4:128-139. https://doi.org/10.1007/ s11764-009-0114-1

7. Galvão DA, Taaffe DR, Spry N, Joseph D, Turner D, Newton RU (2009) Reduced muscle strength and functional performance in men with prostate cancer undergoing androgen suppression: a comprehensive cross-sectional investigation. Prostate Cancer Prostatic Dis 12:198-203. https://doi.org/10.1038/pcan.2008.51

8. Greenspan SL, Coates P, Sereika SM, Nelson JB, Trump DL, Resnick NM (2005) Bone loss after initiation of androgen deprivation therapy in patients with prostate cancer. J Clin Endocrinol Metab 90:6410-6417. https://doi.org/10.1210/jc.2005-0183

9. Shahinian VB, Kuo YF, Freeman JL, Goodwin JS (2005) Risk of fracture after androgen deprivation for prostate cancer. N Engl J Med 352:154-164. https://doi.org/10.1056/NEJMoa041943

10. McGinty HL, Phillips KM, Jim HSL et al (2014) Cognitive functioning in men receiving androgen deprivation therapy for prostate cancer: a systematic review and meta-analysis. Support Care Cancer 22:2271-2280. https://doi.org/10.1007/s00520-014-2285-1

11. Delbaere K, Kochan NA, Close JC et al (2012) Mild cognitive impairment as a predictor of falls in community-dwelling older people. Am J Geriatr Psychiatry 20:845-853. https://doi.org/10. 1097/JGP.0b013e31824afbc4

12. Beaudart C, Zaaria M, Pasleau F, Reginster JY, Bruyère $O$ (2017) Health outcomes of sarcopenia: a systematic review and meta-analysis. PLoS One 12:1-16. https://doi.org/10.1371/journal.pone. 0169548

13. Winters-Stone KM, Moe E, Graff JN, Dieckmann NF, Stoyles S, Borsch C, Alumkal JJ, Amling CL, Beer TM (2017) Falls and frailty in prostate cancer survivors: current, past, and never users of androgen deprivation therapy. J Am Geriatr Soc 65:1414-1419. https://doi.org/10.1111/jgs.14795

14. Garber CE, Blissmer B, Deschenes MR, Franklin BA, Lamonte MJ, Lee IM, Nieman DC, Swain DP, American College of Sports Medicine (2011) Quantity and quality of exercise for developing and maintaining cardiorespiratory, musculoskeletal, and neuromotor fitness in apparently healthy adults: guidance for prescribing exercise. Med Sci Sports Exerc 43:1334-1359. https:// doi.org/10.1249/MSS.0b013e318213fefb

15. Hasenoehrl T, Keilani M, Komandj S et al (2015) The effects of resistance exercise on physical performance and health-related quality of life in prostate cancer patients: a systematic review. Support Care Cancer 23:2479-2497. https://doi.org/10.1007/ s00520-015-2782-x

16. Edmunds K, Tuffaha H, Scuffham P, Galvão DA, Newton RU (2020) The role of exercise in the management of adverse effects of androgen deprivation therapy for prostate cancer: a rapid review. Support Care Cancer. https://doi.org/10.1007/s00520-020-05637-0

17. Keilani M, Hasenoehrl T, Baumann L, Ristl R, Schwarz M, Marhold M, Sedghi Komandj T, Crevenna R (2017) Effects of resistance exercise in prostate cancer patients: a meta-analysis. Support Care Cancer 25:2953-2968. https://doi.org/10.1007/ s00520-017-3771-z

18. Alberga AS, Segal RJ, Reid RG et al (2012) Age and androgendeprivation therapy on exercise outcomes in men with prostate cancer. Support Care Cancer 20:971-981. https://doi.org/10.1007/ s00520-011-1169-x

19. Gardner JR, Livingston PM, Fraser SF (2014) Effects of exercise on treatment-related adverse effects for patients with prostate cancer receiving androgen-deprivation therapy: a systematic review. J Clin Oncol 32:335-346. https://doi.org/10.1200/JCO.2013.49.5523

20. Mundell NL, Daly RM, Macpherson H, Fraser SF (2017) Cognitive decline in prostate cancer patients undergoing ADT: a potential role for exercise training. Endocr Relat Cancer 24:R145-R155. https:// doi.org/10.1530/ERC-16-0493

21. Joseph SJ, Lam V, Patel M (2019) Preventing osteoporosis in men taking androgen deprivation therapy for prostate cancer: a systematic review and meta-analysis. Eur Urol Oncol 2:551-561. https:// doi.org/10.1016/j.euo.2018.11.001

22. Moher D, Liberati A, Tetzlaff J, Altman DG, The PRISMA Group (2009) Preferred reporting items for systematic reviews and metaanalyses: the PRISMA statement. PLoS Med 6:e1000097. https:// doi.org/10.1371/journal.pmed.1000097

23. Higgins JP, Altman DG, Gøtzsche PC et al (2011) The Cochrane Collaboration's tool for assessing risk of bias in randomised trials. BMJ 343:d5928. https://doi.org/10.1136/bmj.d5928

24. Cormie P, Galvão DA, Spry N, Joseph D, Chee R, Taaffe DR, Chambers SK, Newton RU (2015) Can supervised exercise prevent treatment toxicity in patients with prostate cancer initiating androgen-deprivation therapy: a randomised controlled trial. BJU Int 115:256-266. https://doi.org/10.1111/bju.12646

25. Newton RU, Galvão DA, Spry N et al (2019) Exercise mode specificity for preserving spine and hip bone mineral density in prostate cancer patients. Med Sci Sports Exerc 51:607-614. https://doi.org/ 10.1249/MSS.0000000000001831

26. Taaffe DR, Galvão DA, Spry N, Joseph D, Chambers SK, Gardiner RA, Hayne D, Cormie P, Shum DHK, Newton RU (2019) Immediate versus delayed exercise in men initiating androgen deprivation: effects on bone density and soft tissue composition. BJU Int 123:261-269. https://doi.org/10.1111/bju.14505

27. Nilsen TS, Raastad T, Skovlund E, Courneya KS, Langberg CW, Lilleby W, Fosså SD, Thorsen L (2015) Effects of strength training on body composition, physical functioning, and quality of life in prostate cancer patients during androgen deprivation therapy. Acta Oncol 54:1805-1813. https://doi.org/10.3109/0284186X.2015. 1037008

28. Uth J, Hornstrup T, Christensen JF, Christensen KB, Jørgensen NR, Helge EW, Schmidt JF, Brasso K, Helge JW, Jakobsen MD, Andersen LL, Rørth M, Midtgaard J, Krustrup P (2016) Football training in men with prostate cancer undergoing androgen deprivation therapy: activity profile and short-term skeletal and postural balance adaptations. Eur J Appl Physiol 116:471-480. https://doi. org/10.1007/s00421-015-3301-y 
29. Uth J, Hornstrup T, Christensen JF, Christensen KB, Jørgensen NR, Schmidt JF, Brasso K, Jakobsen MD, Sundstrup E, Andersen LL, Rørth M, Midtgaard J, Krustrup P, Helge EW (2016) Efficacy of recreational football on bone health, body composition, and physical functioning in men with prostate cancer undergoing androgen deprivation therapy: 32-week follow-up of the FC prostate randomised controlled trial. Osteoporos Int 27:1507-1518. https:// doi.org/10.1007/s00198-015-3399-0

30. Bjerre ED, Brasso K, Jørgensen AB, Petersen TH, Eriksen AR, Tolver A, Christensen JF, Poulsen MH, Madsen SS, Østergren PB, Borre M, Krustrup P, Johansen C, Rørth M, Midtgaard J (2019) Football compared with usual care in men with prostate cancer (FC Prostate Community Trial): a pragmatic multicentre randomized controlled trial. Sports Med 49:145-158. https://doi. org/10.1007/s40279-018-1031-0

31. Alibhai SMH, Santa Mina D, Ritvo P, Tomlinson G, Sabiston C, Krahn M, Durbano S, Matthew A, Warde P, O’Neill M, Timilshina N, Segal R, Culos-Reed N (2019) A phase II randomized controlled trial of three exercise delivery methods in men with prostate cancer on androgen deprivation therapy. BMC Cancer 19:1-11. https:// doi.org/10.1186/s12885-018-5189-5

32. Newton RU, Kenfield SA, Hart NH, Chan JM, Courneya KS, Catto J, Finn SP, Greenwood R, Hughes DC, Mucci L, Plymate SR, Praet SFE, Guinan EM, van Blarigan EL, Casey O, Buzza M, Gledhill S, Zhang L, Galvão DA, Ryan CJ, Saad F (2018) Intense exercise for survival among men with metastatic castrate-resistant prostate cancer (INTERVAL-GAP4): a multicentre, randomised, controlled phase III study protocol. BMJ Open 8:e22899. https://doi.org/10. 1136/bmjopen-2018-022899

33. Basaria S, Storer T. RESIST-ADT (androgen deprivation therapy). In: clinicaltrial.gov. Accessed 2 Sept 2020

34. Newton RU, Galvão DA, Spry N, Joseph D, Chambers SK, Gardiner RA, Hayne D, Taaffe DR (2020) Timing of exercise for muscle strength and physical function in men initiating ADT for prostate cancer. Prostate Cancer Prostatic Dis 23:457-464. https:// doi.org/10.1038/s41391-019-0200

35. Tsang DS, Jones JM, Samadi O, Shah S, Mitsakakis N, Catton CN, Jeon W, To J, Breunis H, Alibhai SMH (2018) Healthy bones study: can a prescription coupled with education improve bone health for patients receiving androgen deprivation therapy?-a before/after study. Support Care Cancer 26:2861-2869. https:// doi.org/10.1007/s00520-018-4150-0

36. Santa Mina D, Ritvo P, Matthew AG et al (2012) Group exercise versus personal training for prostate cancer patients: a pilot randomized trial. J Cancer Ther 03:146-156. https://doi.org/10.4236/jct. 2012.32020

37. Papadopoulos E, Santa Mina D, Culos-Reed N et al (2020) Effects of six months of aerobic and resistance training on metabolic markers and bone mineral density in older men on androgen deprivation therapy for prostate cancer. J Geriatr Oncol S1879-4068(19): 30378-30379. https://doi.org/10.1016/j.jgo.2020.02.013

38. Alibhai SM, Durbano S, O'Neill M et al (2018) Effects of a 6month moderate-intensity exercise program on metabolic parameters and bone mineral density in men on androgen deprivation therapy for prostate cancer. J Clin Oncol 36:237. https://doi.org/10. 1200/JCO.2018.36.6_suppl.237

39. Alibhai SM (2014) An exercise trial and economic analysis in men with prostate cancer (ADT Ex RCT). In: clinicaltrial.gov. ClinicalTrials.gov. Accessed 7 Feb 2020

40. Wolin KY (2009) A pilot study of physical activity for improving quality of life in prostate cancer patients on androgen deprivation therapy (ADT) (MAP-P). In: clinicaltrial.gov. Accessed 7 Feb 2020

41. Lee CE, Leslie WD, Lau YKJ (2012) A pilot study of exercise in men with prostate cancer receiving androgen deprivation therapy. BMC Cancer 12:103. https://doi.org/10.1186/1471-2407-12-324
42. Brinkman M, Trumpour S, Lyford K, Gray D (2018) Choosing healthy eating choices and keeping active for men having the androgen therapy experience the Checkmate pilot study. BJU Int 122:16

43. Owen PJ, Daly RM, Livingston PM, Mundell NL, Dalla Via J, Millar JL, Fraser SF (2017) Efficacy of a multi-component exercise programme and nutritional supplementation on musculoskeletal health in men treated with androgen deprivation therapy for prostate cancer (IMPACT): study protocol of a randomised controlled trial. Trials 18:1-16. https://doi.org/10.1186/s13063-017-2185-z

44. Georgetown University (2014) An exercise intervention for prostate cancer patients receiving androgen deprivation therapy (MIGHTY). In: clinicaltrial.gov. Accessed 7 Feb 2020

45. Baumann F, Großek A, Hafke R, Wrensch M (2016) Influences of targeted exercise therapy on treatment-related side effects of hormonetherapy in prostate cancer patients. In: Ger. Clin. trial Regist. https://www.drks.de/drks_web/navigate.do?navigationId= trial.HTML\&TRIAL_ID=DRKS00009975. Accessed 7 Feb 2020

46. Winters-Stone K. GET FIT Prostate: a randomized controlled exercise trial. In: clinicaltrial.gov. https://clinicaltrials.gov/ct2/show/ NCT03741335 ? cond $=$ get-fit $\&$ draw $=2 \&$ rank $=6$. Accessed 7 Feb 2020

47. Murtola T (2019) Exercise and quality of life during androgen deprivation therapy. In: clinicaltrial.gov. https://clinicaltrials.gov/ ct $2 /$ show/NCT04050397? cond=Exercise+and+quality+of+Life+ during+androgen+deprivation+therapy $\&$ draw $=2 \&$ rank $=1$. Accessed $7 \mathrm{Feb} 2020$

48. Capela A (2019) Walking football as a supportive medicine for patients with prostate cancer. In: clinicaltrial.gov.https:// clinicaltrials.gov/ct2/show/NCT04062162?cond=Walking+ football+as+a+supportive+medicine+for+patients+with+prostate+ cancer\&draw $=2 \&$ rank $=1$ Accessed 7 Feb 2020

49. Winters-Stone KM, Dobek JC, Bennett JA, Maddalozzo GF, Ryan CW, Beer TM (2014) Skeletal response to resistance and impact training in prostate cancer survivors. Med Sci Sports Exerc 46: 1482-1488. https://doi.org/10.1249/MSS.0000000000000265

50. Kim SH, Seong DH, Yoon SM, Choi YD, Choi E, Song Y, Song H (2018) The effect on bone outcomes of home-based exercise intervention for prostate cancer survivors receiving androgen deprivation therapy: a pilot randomized controlled trial. Cancer Nurs 41: 379-388. https://doi.org/10.1097/NCC.0000000000000530

51. Uth J, Schmidt JF, Christensen JF, Hornstrup T, Andersen LJ, Hansen PR, Christensen KB, Andersen LL, Helge EW, Brasso K, Rørth M, Krustrup P, Midtgaard J (2013) Effects of recreational soccer in men with prostate cancer undergoing androgen deprivation therapy: study protocol for the "FC Prostate" randomized controlled trial. BMC Cancer 13:1-10. https://doi.org/10.1186/14712407-13-595

52. Williams AD, Bird ML, Hardcastle SG, Kirschbaum M, Ogden KJ, Walters JA (2018) Exercise for reducing falls in people living with and beyond cancer. Cochrane Database Syst Rev 2018:. https://doi. org/10.1002/14651858.CD011687.pub2

53. Kohrt WM, Bloomfield SA, Little KD, Nelson ME, Yingling VR (2004) Physical activity and bone health. Med Sci Sports Exerc 36: 1985-1996. https://doi.org/10.1249/01.MSS.0000142662.21767. 58

54. Hernlund E, Svedbom A, Ivergård M et al (2013) Osteoporosis in the European Union: medical management, epidemiology and economic burden: a report prepared in collaboration with the International Osteoporosis Foundation (IOF) and the European Federation of Pharmaceutical Industry Associations (EFPIA). Arch Osteoporos 8(1-2):136. https://doi.org/10.1007/s11657-0130136-1

55. Bonafede M, Shi N, Barron R, Li X, Crittenden DB, Chandler D (2016) Predicting imminent risk for fracture in patients aged 50 or older with osteoporosis using US claims data. Arch Osteoporos 11(1):26. https://doi.org/10.1007/s11657-016-0280-5 
56. Cauley JA, Cawthon PM, Peters KE, Cummings SR, Ensrud KE, Bauer DC, Taylor BC, Shikany JM, Hoffman AR, Lane NE, Kado DM, Stefanick ML, Orwoll ES, for the Osteoporotic Fractures in Men (MrOS) Study Research Group (2016) Risk factors for hip fracture in older men: the osteoporotic fractures in men study (MrOS). J Bone Miner Res 31:1810-1819. https://doi.org/10. 1002/jbmr.2836

57. Tinetti ME, Speechley M, Ginter SF (1988) Risk factors for falls among elderly persons living in the community. N Engl J Med 319: 1701-1707. https://doi.org/10.1056/NEJM198812293192604

58. Tinetti ME, Williams CS (1997) Falls, injuries due to falls, and the risk of admission to a nursing home. N Engl J Med 337:1279-1284. https://doi.org/10.1056/NEJM199710303371806

59. Heinrich S, Rapp K, Rissmann U, Becker C, König HH (2010) Cost of falls in old age: a systematic review. Osteoporos Int 21:891-902. https://doi.org/10.1007/s00198-009-1100-1

60. McGrath R, Al Snih S, Markides K, Hall O, Peterson M (2019) The burden of health conditions for middle-aged and older adults in the United States: disability-adjusted life years. BMC Geriatr 19:1-13. https://doi.org/10.1186/s12877-019-1110-6

61. Kanis JA, Cooper C, Rizzoli R et al (2017) Identification and management of patients at increased risk of osteoporotic fracture: outcomes of an ESCEO expert consensus meeting. Osteoporos Int 28: 2023-2034. https://doi.org/10.1007/s00198-017-4009-0
62. El-Khoury F, Cassou B, Charles MA, Dargent-Molina P (2013) The effect of fall prevention exercise programmes on fall induced injuries in community dwelling older adults: systematic review and meta-analysis of randomised controlled trials. BMJ 347:f6234. https://doi.org/10.1136/bmj.f6234

63. Krupski TL, Foley KA, Baser O, Long S, Macarios D, Litwin MS (2007) Health care cost associated with prostate cancer, androgen deprivation therapy and bone complications. J Urol 178:14231428. https://doi.org/10.1016/j.juro.2007.05.135

64. Bird ML, Cheney MJ, Williams AD (2016) Accidental fall rates in community-dwelling adults compared to cancer survivors during and post-treatment: a systematic review with meta-analysis. Oncol Nurs Forum 43:E64-E72. https://doi.org/10.1188/16.ONF.E64E72

65. Bourke L, Smith D, Steed L, Hooper R, Carter A, Catto J, Albertsen PC, Tombal B, Payne HA, Rosario DJ (2016) Exercise for men with prostate cancer: a systematic review and meta-analysis. Eur Urol 69:693-703. https://doi.org/10.1016/j.eururo.2015.10.047

Publisher's note Springer Nature remains neutral with regard to jurisdictional claims in published maps and institutional affiliations. 